\title{
Gestión para el diseño e implementación de indicadores de atención médica de alta especialidad
}

\author{
SimÓn KaWA-KaRASIK* \\ Director General de Coordinación de los Institutos Nacionales de Salud, Comisión Coordinadora de los Institutos Nacionales de \\ Salud y Hospitales de Alta Especialidad, Ciudad de México, México
}

Para el cumplimiento de los objetivos estratégicos de la Comisión Coordinadora de los Institutos Nacionales de Salud y Hospitales de Alta Especialidad (CINSHAE), la Dirección General de Coordinación de los Institutos Nacionales de Salud (DGCINS) propuso establecer los procedimientos que vincularan la política nacional de salud con los programas federales, sectoriales e institucionales, para garantizar que los institutos y hospitales coordinados proporcionen medicina de alta especialidad acorde con la capacidad instalada y susceptible de ser evaluada mediante indicadores de su complejidad.

Históricamente, la medición del desempeño institucional se ha caracterizado por la valoración cuantitativa y cualitativa del logro de metas programáticas y de la productividad hospitalaria a través de los Informes de Autoevaluación presentados ante las Juntas de Gobierno, con apego a la normatividad y con base en metodologías de gestión para resultados, presupuesto basado en resultados, sistema de evaluación del desempeño y metodología de marco lógico, que sin dejar de ser útiles por la robustez de su diseño, no alcanzan a integrar del todo en su construcción la complejidad del cómo se realizan y quiénes hacen las actividades de la medicina de alta especialidad, conformada por servicios de atención medicoquirúrgica de patologías de baja frecuencia asociadas a comorbilidades que involucran el empleo de una combinación de procedimientos diagnósticos y terapéuticos con tecnología punta de alta precisión y elevado costo, realizados por profesionales en equipos multidisciplinarios.

Por ello, en el año 2015 la DGCINS realizó el Estudio del reporte de indicadores de gestión de medicina de alta especialidad, cuyos resultados mostraron que para subsanar los defectos de estos enfoques se requiere contar con un sistema de indicadores que no se limite a medir el uso eficiente de los recursos públicos ni establecer acciones para la mejora continua de las instituciones mediante la fiscalización, sino que adicione elementos de alineación de recursos, de eficacia, de calidad y de efectividad clínica que, por medio de su análisis, identifiquen los resultados de las intervenciones, el impacto costo-efectividad y en la calidad de vida o en la preservación de la misma, con acciones de alta complejidad.

Del citado estudio se desprendieron las siguientes tres líneas de trabajo, que fueron formalizadas ante la

\author{
Correspondencia: \\ *Simón Kawa-Karasik \\ Comisión Coordinadora de los Institutos Nacionales de Salud \\ y Hospitales de Alta Especialidad \\ Periférico Sur, 4118, Piso 1 \\ Col. Jardines del Pedregal, Del. Álvaro Obregón \\ C.P. 01900, Ciudad de México, México \\ E-mail: simon.kawa.ccinshae@gmail.com
}

Date of reception: 18-06-2018

Date of acceptance: 20-06-2018

DOI: 10.24875/HMCM.M18000003 
Secretaría de la Función Pública mediante un plan para tal efecto, con documentación periódica de los avances y desarrolladas por grupos de expertos institucionales:

1. Redimensionamiento de los indicadores que conforman la matriz de indicadores de resultados (MIR).

2. Desarrollo de indicadores de calidad y seguridad del paciente, de comparabilidad internacional y acorde con la factibilidad organizacional para su instrumentación.

3. Desarrollo de indicadores propios de la medicina de alta especialidad conforme a las actividades sustantivas.

Para su ejecución se incluyó a todas las entidades agrupadas en la CCINSHAE mediante acciones encaminadas a establecer los procedimientos más convenientes para: a) evaluar la gestión y el desempeño; b) analizar el contenido y la calidad de los informes de autoevaluación para, en su caso, poder establecer nuevas reglas de reporte, y c) actualizar el formato del informe de autoevaluación.

En este contexto se tomó la decisión conjunta de elaborar el Procedimiento para el Seguimiento y Evaluación del Desempeño Institucional en los Institutos Nacionales de Salud, el cual ordena y sistematiza la intervención de la DGCINS en la evaluación del desempeño, así como en el Sistema Nacional de Información en Salud, contribuyendo a la obtención de estadísticas de calidad de la prestación de servicios en las unidades médicas de alta especialidad de forma oportuna, comparable y útil para la planeación y toma de decisiones.

Entre tanto, durante el proceso de revisión de los informes de autoevaluación a las Juntas de Gobierno correspondientes a la primera sesión ordinaria del 2015, se instruyó a las entidades coordinadas para alinear sus programas de trabajo a las metas establecidas en el Programa de Acción Específico Medicina de Alta Especialidad (PAEMAE), verificando su integración en la segunda sesión ordinaria del mismo año. Asimismo, se solicitó a los prosecretarios de cada Junta de Gobierno la alineación de sus programas anuales de trabajo a este proyecto institucional.

Operativamente, la primera línea de trabajo se concluyó mediante la inclusión, en la MIR 2016, del Programa
Presupuestario de Atención a la Salud, con los siguientes cuatro indicadores apropiados para la medicina de alta especialidad:

1.Porcentaje de auditorías clínicas incorporadas.

2. Porcentaje de procedimientos diagnósticos ambulatorios de alta especialidad realizados.

3. Porcentaje de procedimientos terapéuticos ambulatorios de alta especialidad realizados.

4. Porcentaje de sesiones de rehabilitación especializadas realizadas respecto al total realizado.

Estos indicadores fueron elaborados conjuntamente por las Direcciones Generales (DG) de Coordinación de los Hospitales Federales (HFR) y de los Hospitales Regionales de Alta Especialidad (HRAE), además de la Dirección de Proyectos Estratégicos, logrando obtener el consenso interno y de entidades evaluadoras de la Secretaría de Salud y otras externas, como el Consejo Nacional de Evaluación de la Política de Desarrollo Social, la Secretaría de la Función Pública y la Comisión Económica para América Latina y el Caribe.

El resultado impulsó la instrumentación de estos indicadores a partir del ejercicio fiscal del 2017, lo que se acompañó del diseño y elaboración del mapa conceptual con la alineación respectiva a los programas sexenales, como el Plan Nacional de Desarrollo, el Programa Sectorial de Salud, el PAEMAE y el Programa Anual de Trabajo.

Para la segunda línea de trabajo, en enero de 2015 la CCINSHAE, con la asesoría del Instituto Nacional de Salud Pública, se avocó al trabajo para la revisión de indicadores de atención médica en la alta especialidad y elaborar una Propuesta de Indicadores para los Institutos Nacionales de salud y otros centros de alta especialidad, que, aún sin concluir, está constituida por dos bloques de ocho indicadores cada uno, con el fin de establecer una base sólida de monitorización en el mejoramiento de la calidad y la comparación de resultados, quedando pendiente el pilotaje correspondiente y el análisis de pertinencia institucional para definir su inclusión como elementos de medición acordes con la realidad organizacional.

En cuanto al cumplimiento de la tercera línea de trabajo, se inició con la propuesta de diseñar indicadores 
apropiados para la medición del desempeño institucional en cinco áreas clínicas de las entidades agrupadas en la CCINSHAE: hospitalización, cirugía, áreas críticas, atención ambulatoria y control de infecciones intrahospitalarias.

Para tal fin, bajo la coordinación general y adjunta de los titulares de la DGCINS y de la DGCHRAE, respectivamente, se creó el Grupo Directivo de Indicadores de Medicina de Alta Especialidad, constituido por los Directores Médicos y de Operaciones de los INSalud, los HFR y los HRAE, organizados en cinco subgrupos de trabajo.

Así se validaron y aprobaron 82 indicadores, resultantes de la revisión realizada a la información de atención médica reportada en el Informe de Autoevaluación para Junta de Gobierno, incluidos los ocho indicadores de la Propuesta de indicadores para los Institutos Nacionales de Salud y otros centros de alta especialidad, que solventaron las observaciones derivadas de su pilotaje institucional, así como los cuatro integrados a la MIR del Programa Presupuestario de Atención a la Salud del año 2016.

Los resultados de las tres líneas de trabajo planteadas en líneas anteriores confluyeron en el Catálogo de Indicadores de Atención Médica de Alta Especialidad, inicialmente para su comprensión ejecutiva mediante un listado por servicios y una carta descriptiva. Secuencialmente, para su instrumentación operativa mediante la ficha técnica de cada indicador y los formatos para la captura de información y el cálculo automatizado.

En este encuadre, se ha dejado espacio para que el ejercicio de monitoreo bajo indicadores por parte de las unidades coordinadas ajuste ciertos indicadores de acuerdo a las condiciones del paciente (complejidad de atención y comorbilidad) $)^{1,2}$ y características de las instituciones, de tal forma que su utilización permita comparaciones espaciotemporales, e inter-intra hospitales o servicios hospitalarios ${ }^{3}$.
En cuanto al impacto de los resultados de la medición en el aumento en la calidad de la atención, se debe considerar que la implementación de un sistema de indicadores de gestión no tiene un carácter punitivo, dado que debe estar mediada, como en este caso, por compromisos de gestión programados y establecidos con antelación entre los encargados de la medición y los responsables de la ejecución de las medidas de corrección.

Esto bajo la consideración de que no es suficiente con aplicar un sistema de indicadores y evaluarlos, hay que hacer devoluciones de resultados e implementar otras estrategias de educación, bajo modelos específicos, a los directivos de los hospitales ${ }^{4,5}$.

Es importante mencionar también que los informes de gestión hospitalaria que alimentan a los indicadores deben ser emitidos y publicados por cada hospital de una manera uniforme y con el consenso de que lo que se mide son actividades de procesos y procedimientos clave para el desempeño, definidas por el nivel de resolución y la vocación institucional de atención médica, de modo que los interesados puedan comenzar a hacer preguntas en cuanto a la gestión y la eficiencia ${ }^{3}$.

Así es posible evitar la inoperancia de indicadores que actúan en detrimento del desempeño de las unidades de alta especialidad, cuyo derrame estadístico debe tener cabida específica en los sistemas de información en salud nacionales.

\section{BIBLIOGRAFÍA}

1. Shahian D, Normand Sh. Comparison of risk-adjusted hospital outcomes. Circulation. 2008;117:1955-63.

2. Li B, Evans D, Faris P, Dean S, Quan H. Risk adjustment performance of Charlson and Elixhauser comorbidities in ICD-9 and ICD-10 administrative databases. BMC Health Serv Res. 2008;8:12.

3. Morera-Salas M. Revisión sistemática de indicadores de desempeño hospitalario. Acta Médica Costarricense. 2013;55:75-6.

4. De Vos M, Graafmans W, Kooistra M, Meijboom B, Van Der Vort P, Westert G. Using quality indicators to improve hospital care: a review of the literature. Int J Qual Health Care. 2009;21:119-29.

5. Gibberd R, Hancock S, Howley P, Kay R. Using indicators to quantify the potential to improve the quality of health care. Int J Qual Health Care. 2004; $16: i 37-43$ 